\title{
Lograr la equidad en educación a través de competencias interculturales e intersociales
}

\author{
Achieving Equity in Education through Intercultural and Inter- \\ social Competences
}

\author{
Pablo Santaolalla Rueda \\ Universidad Veracruzana Intercultural \\ Instituto Investigaciones Educativas
}

Recibido: 19/08/2019

Aceptado: 27/11/2019

\begin{abstract}
During a research carried out for 6 years between Spain and Mexico, we have been observing five Secondary schools. In all these multicultural educational centers, we observed the phenomenon about 'failure' and/or educational 'success' and how students overcome (or not) Middle School. Carried by an Implied-Applied Research (Santaolalla, 2016a), we questioned in what grade the cultural and the social class phenomenons impact on this 'overcoming'. From an initial participative observation, a first concern arises: knowing that the five schools (public, private, at the 'center' and the 'periphery' of the city, 'rich' and 'poor', ...) are multicultural, Why Educational systems works in some schools and does not in others? If it is not exclusively related to multiculturality, there must be another impact factor. Result shows that, although multiculturalism is a mandatory aspect to be taken into account in educational action plans, we are forgetting about another key factor: social class (as it operates as a segregation system). Focusing in inclusion, equity and social justice, the present article aims to show how the teaching-learning process needs to provide Intercultural and Intersocial Competences as the backbone of Education to answer the present and future needs.
\end{abstract}

KEY WORDS: Intersocial Education; Intercultural Education; Equity; Educational Models; Applied-Implied Research; Education in marginalized contexts; Intercultural Competences; Intersocial Competences.

\begin{abstract}
RESUMEN
Durante una investigación llevada a cabo durante 6 años entre España y México, hemos observado cinco centros educativos. En todos los centros, multiculturales, observamos el fenómeno sobre el fracaso y/o éxito educativo y sobre la superación de la etapa secundaria por parte del alumnado. Realizada una Investigación Aplicada Implicada (Santaolalla, 2016a), nos preguntamos en qué grado el fenómeno cultural y de clase social repercuten sobre dicha superación. Desde la observación participativa inicial, surgía una primera inquietud: sabiendo que los cinco centros (públicos, privados, del "centro" y la "periferia" de la ciudad, "ricos" y "pobres") son multiculturales, ¿por qué en unos funciona el sistema educativo y en otros no? Si no es referente exclusivamente a dicha multiculturalidad, debe haber otro factor de incidencia. El resultado muestra que, si bien la multiculturalidad es un aspecto esencial a tener en cuenta en los planes de actuación educativos, es decir, las competencias interculturales, nos estamos olvidando de otro factor clave: la clase social (que opera como sistema de segregación). Centrando su enfoque en la inclusión, equidad y justicia social, el presente artículo pretende mostrar cómo la Educación debe vertebrarse en la enseñanza de competencias interculturales e intersociales, para dar respuesta a las necesidades del presente y para el futuro. PALABRAS CLAVE: Educación Intersocial; Educación Intercultural; Equidad; Modelo Educacional; Investigación Aplicada Implicada; Educación en contextos marginados; Competencias interculturales; Competencias Intersociales.
\end{abstract}

Autor de correspondencia

Pablo Santaolalla Rueda, Universidad Veracruzana Intercultural-Instituto Investigaciones Educativas, Universidad Miguel Hernández. E-mail: pablosantaolallarueda@gmail.com ORCID: 


\section{Escuelas diversas en diversos lugares}

Vemos en la actualidad, y lo llevamos viendo desde hace décadas, que las escuelas —lugares de convivencia - se convierten en verdaderos campos de batalla (Santaolalla, 2016b). El alumnado, el profesorado, las familias, el vecindario: todo aparece disgregado. Se vive, pero no se convive. Todo ello parece acrecentarse en barrios obreros (en el caso de España), en las culturas urbano-populares mexicanas — perfectamente definidas por Bonfil Batalla (1995) —, en zonas 'deprimidas' de la ciudad, en los llamados 'guetos', en la 'periferia', en zonas en 'riesgo de exclusión social'. Y he aquí donde surge la cuestión de por qué, si se habla de equidad, se ha propuesto un mismo modelo globalizado, homogéneo, con ciertas características estéticas y cosméticas, donde no se tiene en cuenta dicha diversidad cultural y social.

En el texto nos referiremos a diversidad cultural (Mato, 2008) como objetivo a lograr. En el trabajo citado se recogen un total de 38 trabajos acerca de dicho término desde la óptica y experiencia en 11 países que compartimos en cuanto al sentido. Entendida como multiculturalidad (puede o no ser un hecho intercultural), pero también entendiendo la intraculturalidad (Santaolalla, 2016a) del propio alumnado (cultura familiar, de origen, cultura juvenil, cultura del micro y macrocontexto, cultura escolar, etc.). Hablaremos de ella como objetivo, puesto que la entendemos como la legitimación de las diferencias culturales entre diversos grupos humanos y ya que a su vez evoca una convivencia donde se promuevan relaciones interculturales provechosas. Para el presente trabajo, en específico, la diversidad cultural se trabaja desde el ámbito de diversidad de países de procedencia del alumnado — aunque esto reduzca el sentido completo y complejo de dicho término- y/o sus familias, así como la importancia (nivel de incidencia) que esta diversidad representa en los centros educativos como factores que pueden incidir en la superación de la etapa Secundaria.

Este hecho de la 'igualdad', como hemos observado en la presente investigación, deriva en desigualdad (paradójicamente), que es lo mismo que hablar de injusticia social. Explicar el por qué y el cómo nos sirve para visibilizar las nuevas formas de segregación y, por tanto, de hallar un camino desde el que actuar. Si en lugar de igualdad hablamos de equidad en educación, automáticamente implica diversidad, de forma que se pueda/deba reconocer las circunstancias que generan la cultura y clase social en el alumnado. En el caso concreto del presente estudio, al hablar de diversidad cultural lo haremos para reconocer los diferentes países de procedencia, pero, igualmente, para legitimar el concepto de equidad educativa como necesidad aplicable a esto.

Otro tema imperativo para el objeto de estudio era hablar sobre las competencias. En el trabajo observamos la necesidad de educar en competencias interculturales e intersociales. Nos inclinamos a definiciones de competencias interculturales desde Barret (2013), a cómo llevaras a la práctica (Byram, 2001) y los desafíos que ésta nos traen en el mundo globalizado (Portera y Grant, 2017). Como bien recoge Gunther Dietz (2019):

[...] desde hace aproximadamente dos décadas el conjunto de conocimientos, habilidades, destrezas, actitudes y valores que intervienen en un determinado saber-hacer específico es redefinido y conceptualizado bajo el concepto de competencia (Moya Otero \& Luengo Horcajo, 2011). A diferencia de las anteriores definiciones un tanto fragmentadas de cada uno de los elementos de este conjunto -acerca de lo que es una habilidad, lo que es una destreza, lo que es una actitud, etc.-, la noción de competencia hace énfasis en dos aspectos medulares: en primer lugar, el hecho de que se trata de procedimientos no mecánicos o aditivos, sino complejos y simultáneos en los que interviene a la vez el mencionado conjunto de elementos cognitivos, metodológicos y axiológicos; y, en segundo lugar, el hecho de que este conjunto de sabereshaceres es plasmable, demostrable y evaluable en la práctica concreta, en el hacer y no en algún conocimiento abstracto, memorístico o enciclopédico.

Ahora bien, la pregunta que nos planteábamos a la hora de describir dichas competencias interculturales como necesarias para formar un currículo educativo basado en la equidad, era si sólo debían ser nombradas como tal, como interculturales, o si se debía añadir otro factor al fenómeno multicultural. A través de una comparativa de centros educativo y de la propuesta de tres ejes 
fundamentales: a) diversidad cultural (por país de procedencia); b) diversidad social (por clase social); y c) porcentaje de superación de la etapa educativa de nivel Secundaria, hemos podido comprobar que todos los anteriores se relacionan y cómo ambos factores (a y b) repercuten sobre el éxito o fracaso escolar. A partir de aquí, podremos observar las necesidades actuales en materia de equidad. El resultado de la investigación muestra cómo el factor de clase social es fundamental para entender los procesos educativos, ya que es determinante en la superación de la etapa secundaria; por lo que debemos entender también que se requieren de competencias intersociales para formar un currículo educativo equitativo.

\section{Metodología para una Investigación Aplicada Implicada}

El trabajo que presentamos nace de una investigación previa realizada y que se soporta en tres textos fundamentales para conocer la trayectoria (Santaolalla 2016a, 2016b, 2018). A modo de resumen - para el correcto entendimiento del presente- dicha investigación nace en España, en la provincia de Alicante, y se extiende a México, en la ciudad de Playa del Carmen. La elección de dichas localidades se basa en ser dos ciudades altamente turísticas, con un fuerte índice de habitantes emigrados de otros lugares del globo y similar forma de expansión (crecimiento de las ciudades) a causa del turismo mencionado. Concretamente, el trabajo se recoge en 5 centros educativos escogidos de acuerdo con:

1.Enclave geográfico/cartográfico en la ciudad: zona centro, zona periferia. Para lo cual se realizó un trabajo de campo de 4 años en el caso de España y 2 años en México.

2.Perfil económico: barrio clase obrera, colonia popular, barrio clase media-alta. Esto se resolvió con el análisis previo dentro del trabajo de campo, donde se observó: el nivel económico del precio de vivienda acudiendo al Instituto de la Vivienda de Alicante y al Instituto Nacional de Estadística Geográfica e Informática en México, concretamente en el Gobierno del Estado de Quintana Roo y el Ayuntamiento de Solidaridad y la observación de precios de alquiler y venta de cada zona; entrevistas al vecindario. El tipo de trabajos del vecindario, observado esto a través de la etnografía realizada (comentada abajo).

3. Análisis histórico-etnográfico del crecimiento de cada enclave.

4.Multiculturalidad: heterogeneidad de países de procedencia (nacionalidades) del alumnado y de las familias del alumnado de acuerdo con las bases de registro de cada centro y de un conteo personal (entrevista sencilla) donde se preguntó por varios datos (edad, país de origen y/o procedencia familiar, sexo, religión, trabajo y salario (a las familias) y años viviendo en el contexto actual. De dicho primero contacto (entrevista rápida), se seleccionó la muestra para realizar entrevistas en profundidad, que fueron un total de 178 en el caso de España y 124 en el caso de México. La metodología empleada (Santaolalla, 2016a, pp. 72-73) para las entrevistas personales (a alumnado, profesorado, familias y vecindario), así como algunas transcripciones de entrevistas, aparecen en el trabajo que dio inicio a todo este proceso (Santaolalla, 2016a). El modelo de entrevista en México fue el mismo, ya que el trabajo es una continuación del anterior.

5.Porcentaje de alumnado egresado de secundaria y calificaciones. Esto se trabajó consultando las estadísticas de la Consellería de Educación, Cultura y Deporte de la Comunidad Valenciana (2014) bajo petición expresa y personal en la institución con sede en Alicante y el Instituto Nacional de Evaluación Educativa de España (2014) y los datos del Sistema Nacional de Información y Estadística Educativa de la Secretaría de Educación Pública del Gobierno de México (2017) y Servicios Educativos de Quintana Roo (2017 y 2018) bajo petición personal ante la institución según formularios online en la página, y consultado los datos internos de cada centro (pedidos a la Dirección y la Secretaría de cada centro educativo) en el transcurso de la última década (2009-2019) y el promedio (calificaciones) de los ciclos lectivos actuales a la fecha en la que se realizó cada trabajo de campo en los lugares específicos. 
La observación participativa mencionada, enmarcada como herramienta dentro de la metodología de Investigación-Acción Participativa (IAP) y una Investigación Aplicada Implicada, en adelante IAI (Santaolalla 2016b; 2018), se produce viviendo durante 18 años en el barrio de Juan XXIII (lugar de nacimiento y como alumno y profesor/investigador del I.E.S Las Lomas durante 6 años); 3 años en el barrio del centro de la ciudad de Alicante (lugar de enclave del I.E.S Miguel Hernández, también como profesor durante 2 años y como investigador 1 año); 1 año como investigador en el I.E.S Liceo Francés; 2 años de posdoctorado, uno viviendo en el centro de la ciudad de Playa del Carmen, donde se enmarca la Comunidad Educativa El Papalote, y otro año viviendo en la Colonia Colosio, a la que se adscribe el Centro Educativo José España Cruz. En ambos centros siendo docente e investigador (turno matutino y vespertino, respectivamente).

En los trabajos previos (Santaolalla, 2016a; 2016b) se muestra una etnografía de la ciudad de Alicante y de los barrios (contexto social, cultural, demográfico, económico, etc.) donde se adscriben los centros educativos; además de un análisis en profundidad de uno de los centros educativos (I.E.S Las Lomas) puesto que ahí se centra la tesis doctoral surgida. En el presente hacemos —aunque más breve- un análisis de la Ciudad de Playa del Carmen, para contextualizar la investigación en ambos lugares. Esto sugiere que se consulte el trabajo arriba mencionado si se desea profundizar en los contextos sociales.

Por tanto, la metodología empleada para el estudio global la denominamos IAI (Santaolalla, 2016b, 2018; Bauman, et. al., 2018) y ésta contiene lo descrito anteriormente mediante el empleo de la observación directa y participativa, entrevistas personales y charlas informales, experiencia en los centros educativos (Santaolalla, 2016, pp. 230-277) y en los contextos sociales (trabajo de campo), documentación y análisis de datos científicos, demográficos, etc., que dieron lugar a etnografía (Santaolalla, 2016, pp. 88-105) y al total de los trabajos previos y actuales mencionados.

\section{Interculturalidad e Intersocialidad}

En el barrio de Juan XXIII de Alicante -donde se produjo el primer estudio- viven varias culturas: dos internas, como son la paya y la gitana, a las que desde hace poco menos de dos décadas se añaden en gran cantidad la árabe (principalmente, de Marruecos y Argelia), la senegalesa, la colombiana y la rumana y en distintas proporciones otras de múltiples puntos geográficos del globo (Santaolalla, 2016a). En la ciudad de Alicante, o si nos vamos a la provincia al completo, sucede lo mismo, pero además se le añaden otras culturas y/o grupos sociales que vienen de los mismos países y de otros situados en el centro y norte de Europa (Ministerio de Trabajo, Migraciones y Seguridad Social, 2014; Instituto Nacional Estadística España, 2014; Santaolalla, 2017). ¿En qué difiere, pues, el entorno? La provincia al completo es un espacio multicultural (todavía no podemos hablar de interculturalidad), pero existe una distancia entre los contextos sociales, ergo dicha separación no podemos encontrarla únicamente en el fenómeno multicultural como agente diferenciador. La distancia se observa en el dónde y cómo se distribuyen las distintas culturas. Unas, las del primer caso del barrio de Juan XXIII, vienen por unos motivos muy diferentes a las razones que motivan las migraciones del segundo tipo. Unas (migración forzada) llegan a España por trabajo, huyen de unas condiciones sociales, políticas, económicas, etc., que imposibilitan la vida. Vienen con recursos escasos y llegan a la ciudad con menos de esos recursos, si es que arriban con algo. Las otras, culturas occidentales — similar, pero distinto a la migración voluntaria de la que hablan Ogbu y Simons (1998) — vienen, principalmente, por el clima, por el bajo precio de las viviendas en plena costa y por la cercanía a sus países de origen. El factor que separa aquí es un factor de capital, de la cantidad de recursos económicos — de plusvalías obtenidas y acumulación de riqueza - con los que llegan a su nuevo destino.

La ciudad mexicana se presenta igualmente como un entorno multicultural y arraigada al turismo, pero de población flotante (Panaia, 2010) e itinerante en el sentido de que se han desplazado hacia ella dependiendo de los flujos económicos (Borja y Castells, 1998); pasando de un pueblo de pescadores con apenas 2000 habitantes en los 70-80, a más de 220.000 personas (sin contar las no 
registradas), de acuerdo a los datos que nos ofrecieron en el Palacio Municipal (Ayuntamiento de Playa del Carmen) y al censo del consultado mediante entrevista personal con el Director de Catastro del municipio de Solidaridad. Esto, según las entrevistas personales a 65 vecinos/as de cada zona y a la entrevista con el titular de la Oficina de representación del Instituto Nacional de Inmigración en Quintana Roo citado en Cancún, debido a:

1. Migraciones internas desplazadas de territorios rurales donde ya no existe oportunidad de trabajo (migrantes forzados) y una gran parte de migraciones internas voluntarias de una clase social alta que ven en la Riviera Maya una oportunidad de distinguirse.

2. Migraciones de otros países, principalmente EEUU, Canadá y Europa, y de otros países del continente sud-americano. La diferencia, por un lado, radica en que el migrante del Norte del globo viene, aunque sea a trabajar, en calidad de una categoría pseudo-turística donde vive, trabaja y cobra experiencia sobre la ciudad desde un plano casi ajeno a ésta (migración voluntaria). Por otro lado, el migrante de países del Sur, que viene en busca de trabajo, se asemeja más al núcleo de personas desplazadas desde el interior del país (migrantes forzados), su nivel económico es menor y, por tanto, su lugar de residencia es el mismo que el de las familias mexicanas urbano-populares. Esto no ocurre de igual forma si la persona que migra desde estos países del sur es de clase social media-alta o alta.

De todo ello resumimos una idea: el habitante de Playa del Carmen —así como ocurre con la ciudadanía de Alicante- se reúne y convive (si lo hace) más con aquellas personas con quienes tiene un nivel-estatus económico similar; es decir, prevalece no la cultura o el origen étnico en las disposiciones de grupos de convivencia, sino la clase. Se puede consultar un amplio estudio acerca de las características propias del barrio de Juan XXIII (España) y de la ciudad de Alicante en los estudios previos publicados (Santaolalla, 2016a y 2016b).

Los tres centros ${ }^{39}$ donde realizamos la observación inicial, siendo muy diferentes entre sí, coinciden en ser entornos multiculturales (en base a la heterogeneidad de países de procedencia del alumnado). ¿Qué los difiere? No es el modelo educativo, puesto que los tres quedan sometidos a la Ley de Educación española vigente, salvo ciertas variaciones en el currículo. Lo mismo sucede en en México.

De acuerdo con el trabajo de campo, las entrevistas personales y las fuentes consultadas arriba comentadas, en el caso A, la población es la del primer tipo descrito: paya, gitana, árabe, senegalesa, latinoamericana, rumana, etc. (autóctonos y migrantes forzados). Todos tienen una característica común que viene representada desde sus recursos económicos monetarios (son de clase obrera y/o pobre). En el B, encontramos un alumnado similar, aunque no tan diverso. Su población es, mayormente, española paya y, en menor cantidad, árabe, asiática, latinoamericana, etc. (migrantes pseudo-voluntarios). La diferencia radica en el entorno y los recursos. Ambos son centros públicos y la adjudicación de plazas se establece por personas que viven en el barrio al que pertenece el centro educativo. Las familias árabes entrevistadas que viven en el centro, por ejemplo, vinieron con mayor cantidad de recursos económicos y pudieron, en la mayoría de casos, abrir sus propios negocios. No huían tanto de la pobreza, sino hacia la seguridad. El precio del alquiler o compra de vivienda es bastante mayor (hasta 4 veces, según el Servicio Territorial de Vivienda y Rehabilitación del Ayuntamiento de Alicante) y los datos personalmente recabados mediante mateo de los contextos) que en el barrio de Juan XXIII, pero pudieron acceder a él. Siguen siendo clase obrera, sí, pero con una tendencia más hacia la clase media. En el C, por ser privado (y uno con los precios de matrícula y mensualidades más altos de la ciudad), encontramos, principalmente, alumnado cuyo origen familiar es de países del centro y norte de Europa (migración voluntaria).

39 A) El Instituto de Educación Secundaria (I.E.S.) Las Lomas (público, en el barrio de Juan XXIII, en la 'periferia' de la ciudad); B) I.E.S. Miguel Hernández (público, localizado en el 'centro' de la ciudad); y C) Liceo francés (privado, en las afueras de la ciudad). 
De la información recabada siguiendo la metodología mencionada durante la continuación de la investigación en México, vemos que: A) El Centro Educativo Juan España Cruz (escuela de gobierno), es un centro multicultural donde no se trabaja desde la interculturalidad. Su población es, mayormente, mexicana (migrantes forzados de otros estados del interior de la república), pero también hay una parte de población que procede (al menos en origen familiar) de otros países del sur del continente americano (también migrantes forzados). Como ocurría en el caso A de España, la población es, en su totalidad, o pobre o de clase urbano-popular (equivalente en términos económicos, de trabajo y acceso a recursos a la case obrera española, ya que la clase obrera mexicana es entendida de diferente forma (Batalla, 1995). En el turno vespertino (el observado) de 271 alumnos/as, 10 son de 5 países extranjeros. En el caso de México, no encontramos B) El segundo centro, la Comunidad Educativa El Papalote (privado), presenta datos muy distintos: su población, en lo que se refiere a procedencia de diferentes países, es más heterogénea. De un total de 145 alumnos/as, encontramos 43 que son de origen familiar mexicano, el resto procede de 22 países (Estados Unidos, Canadá, Belice, Cuba, Venezuela, Colombia, Brasil, Chile, Argentina, Uruguay, España, Francia, Italia, Alemania, Países Bajos, Gran Bretaña, Bélgica, Dinamarca, Suiza, Noruega, Suecia y Australia). La diversidad cultural (medida únicamente en estos casos, aunque estamos simplificando, como decíamos, de acuerdo con el país de procedencia) es, por tanto, mayor en este segundo caso.

Si atendemos a los resultados académicos (\% de superación de la etapa secundaria), observamos una nueva característica que nos ayuda a resolver la cuestión planteada:

1. Caso A de España (AE): un $90 \%$ de alumnado no supera la secundaria.

2. Caso B de España (BE): el 5\% no supera la secundaria.

3. Caso $C$ de España (CE): existe un $0 \%$ de no superación.

4. Caso A de México (AM): un 85\% no supera la secundaria.

5. Caso B de México (BM): el porcentaje que no supera la secundaria es igual a cero.

Tenemos tres objetos de observación que extraer desde los datos mencionados: primero, la diversidad cultural por procedencia de distintos países; segundo, la clase social; y tercero, el porcentaje de alumnado que no supera la etapa secundaria. La Tabla 1 resume los datos hasta ahora recogidos:

Tabla 1

Datos generales de los centros educativos observados en España y México

\begin{tabular}{|c|c|c|c|c|c|c|}
\hline \multicolumn{2}{|c|}{ Centro educ ativo } & Contexto & Tipo & $\begin{array}{c}\text { Div. Cultur al por } \\
\text { país de } \\
\text { procedencia }\end{array}$ & Clase social & \multirow{2}{*}{$\begin{array}{c}\begin{array}{c}\% \text { no } \\
\text { superación } \\
\text { etapa } \\
\text { secundaria }\end{array} \\
90 \%\end{array}$} \\
\hline Ae & Las lomas & $\begin{array}{l}\text { Alicante, españa } \\
\text { Periferia de la ciudad }\end{array}$ & Público & 18 países & Obrera - pobre & \\
\hline $\mathbf{B e}$ & $\begin{array}{c}\text { Miguel } \\
\text { hernández }\end{array}$ & $\begin{array}{l}\text { Alicante, españa } \\
\text { Centro de la ciudad }\end{array}$ & Público & 8 países & $\begin{array}{c}\text { Media - } \\
\text { Media/ alta }\end{array}$ & $5 \%$ \\
\hline $\mathrm{Ce}$ & Liceo francés & $\begin{array}{c}\text { Alicante, españa } \\
\text { Urbanizaciones } \\
\text { privadas }\end{array}$ & Privado & 9 países & $\begin{array}{l}\text { Media/alta - } \\
\text { Alta }\end{array}$ & $0 \%$ \\
\hline Am & $\begin{array}{c}\text { José españa } \\
\text { cruz }\end{array}$ & $\begin{array}{c}\text { Playa del carmen, } \\
\text { méxico } \\
\text { Periferia de la ciudad }\end{array}$ & Público & 6 países & $\begin{array}{l}\text { Obrera - } \\
\text { Pobre }\end{array}$ & $85 \%$ \\
\hline $\mathbf{B m}$ & El papalote & $\begin{array}{l}\text { Playa del carmen, } \\
\text { méxico } \\
\text { Centro de la ciudad }\end{array}$ & Privado & 22 países & $\begin{array}{l}\text { Media/alta - } \\
\text { Alta }\end{array}$ & $0 \%$ \\
\hline
\end{tabular}


Los datos representados han sido extraídos de la consulta de datos de matrícula oficiales de cada centro educativo, las entrevistas personales a alumnado y familias de cada centro y contexto social, y los distintos organismos mencionados en el primer apartado cuyas referencias aparecen al final del presente artículo.

Para lo que procede, haremos uso de la anterior tabla desde las tres últimas columnas (div. cultural por país de procedencia y/o clase social como factores que influyen en el \% de no superación de la etapa secundaria). De acuerdo con esto, observamos:

1. Primer objeto de observación (diversidad cultural por país de procedencia): la similitud habida entre el caso AE y el BM, así como la similitud entre los centros BE, CE y AM.

2. El segundo objeto (clase social): la similitud habida entre los centros AE y AM, así como la habida entre los centros BE, CE y BM. Una diferencia claramente observable, por tanto, es que el centro BE ya no comparte características similares con el centro AE o el BM (como ocurría en países de procedencia) pero sí con el CE y el BM.

3. El tercer objeto (porcentaje de alumnado que no supera la secundaria): de nuevo, observamos la similitud entre los centros AE y el AM y las similitudes entre los centros BE, CE y BM; al igual que ocurría con el objeto observado de clase social.

4. El porcentaje de superación de la etapa secundaria está más relacionado con la procedencia de clase social que con la procedencia cultural (entendida aquí únicamente como cultura por país de procedencia, lo cual no sugiere que esto sea lo único que define la cultura, como plantearía Gerd Baumann (2001) en su libro El enigma multicultural).

Desde el amplio trabajo de Antonio Gramsci — y de "autores autorizados en la obra de Gramsci, como Umberto Cerroni, Norberto Bobbio, Cesare Luporini, Nicola Matteuci, María A. Macciocchi, Angelo Broccoli, Perry Anderson, Joseph W. Femia y Paul Ransom" (Camacho, 2006, p. 15) - se describe el análisis sociopolítico de las formas complejas en que se manifiesta el poder trabajo también descrito con extensión por Michel Foucault- como hegemonía ideológica de alcance cultural en formaciones sociales concretas. Desde esto, y como se veía en un trabajo anterior (Santaolalla, 2016a), el sistema educativo viene dado desde el gobierno de un país, que a su vez viene representado desde una clase social (hegemónica: capitalista, alta, económicamente rica, excluyente), en la mayoría de casos, y el modelo de escuela derivado de una cultura determinada (occidental, blanca, opresora) como sistema de reproducción hegemónico (Bourdieu y Passeron, 1977; Baudelot y Establet, 1976; Lerena, 1975; Torres, 1994; Rivero, 2002; Enrico, 2008). Pero este sistema-modelo educativo bancario (Freire, 1973) ha conseguido establecerse en cualquier rincón del globo y, por ende, si bien sigue procediendo desde la cultura hegemónica, el sistema educativo ahora viene dado desde cualquier gobierno, que representa a las clases sociales altas y penetra violentamente (Willis, 1978) en las demás clases, independientemente de en qué contexto cultural nos encontremos. Sigue habiendo un eje cultural determinante, pero la cultura, por sí misma, aunque pueda ser la misma, se encuentra determinada por la clase social. Lo observado durante el trabajo de campo, en el proceso etnográfico, ha sido que, por ejemplo, un árabe marroquí que vive en el barrio de Juan XXIII es similar - culturalmente hablando- a un árabe de Dubái que vive en el centro de la ciudad, pero socialmente hablando, el árabe marroquí se encuentra más próximo al español de Juan XXIII que al árabe rico de Dubái. De igual forma, el árabe rico de Dubái se encuentra más próximo (entorno social, recursos, acceso a vivienda, trabajo, espacios simbólicos, etc.) al español del 'centro' de la ciudad que al árabe marroquí de un barrio de la 'periferia'. Los espacios donde conviven son distintos, lo cual queda determinado por la clase social y, por tanto, también determina los contextos educativos donde van a estudiar. El estudiante español de Las Lomas está más próximo —en la dicotomía del éxito/fracaso escolar - al estudiante mexicano del José España Cruz que al estudiante español del Miguel Hernández o del Liceo Francés, aunque estén en la misma ciudad. Culturalmente hablando, 
no ocurre así, pero en cuanto a la clase social, sí: un ejemplo de la influencia de la clase social sobre el resultado educativo.

Trabajar desde la interculturalidad en entornos multiculturales es imprescindible, pero trabajar sólo desde este objeto de actuación es insuficiente puesto que no abarcamos la amplitud de diversidad de diversidades habidas en la sociedad actual. La prueba está en que, si sólo fuese un aspecto cultural, en entornos monoculturales o de escasa diversidad cultural — como lo era España hace no más de veinte años y como lo era el I.E.S. Las Lomas, por ejemplo, hace tan sólo 15 años-, el fenómeno del fracaso escolar sería algo nuevo, venido con la llegada de inmigrantes y de otras culturas. Estaríamos insinuando que la causa del fracaso escolar es la multiculturalidad y creeríamos que solucionando esto se acabaría el problema. Pero vemos que se hace imprescindible derribar dichos muros que separan lo cultural y lo social a través de una educación intercultural e intersocial que, por un lado, valore la diferencia como elemento que hace único al ser humano y, por otro, que reconozca la igualdad en la diversidad como única vía hacia la convivencia, la justicia, la libertad y la equidad educativa y social.

\section{Conclusiones}

Debemos hablar de la cultura como origen y de la clase social, también desde la categoría de agente cultural, en cuanto a factores que existen y determinan sobre el éxito o fracaso escolar. De hecho, una de las primeras conclusiones a las que hemos llegado tras analizar los entornos educativos sabiendo la diferencia y las equidistancias de los entornos-, ha sido la de que, en primera instancia, encontrándonos en entornos multiculturales, sea la clase social la que procura que el arquetipo hegemónico permee en la educación y disponga de forma prescriptiva (Dietz, 2011) los espacios donde cada actor va a establecerse: segregación física/simbólica.

En el espacio de trabajo que desarrollamos en la actualidad, como director de un centro educativo privado en Playa del Carmen, donde se actúa a la vez como investigador junto con la Escuela General Edmundo Rodríguez Villalva, hemos propuesto una serie de actuaciones enmarcadas como competencias intersociales, además de las competencias interculturales. En total, durante el último año se han trabajado un total de 28 proyectos intersociales entre 4 escuelas (privadas y públicas). De ello están surgiendo propuestas didácticas como transferencia del estudio. Estas propuestas —al igual que el presente texto- se basan en el reconocer los ámbitos necesarios de trabajo para una escuela equitativa del presente y para el futuro. No es el principio fundamental decidir desde la observación y la participación directa si es la diversidad cultural o la de clase social la que más incide en los paradigmas de «éxito» o «fracaso» escolar, ni tampoco suponer que podemos reducir la cultura a factores geográficos. Fundamentalmente, el principal objetivo es hacer notoria la necesidad, como explicábamos, de un modelo educativo que requiera de diversos modelos educativos donde se trabaje lo intercultural e intersocial como ejes vertebradores. Un modelo discursivo que se crea, recrea, genera y se reconoce desde el discurso plural.

Adonde nos lleva todo esto es a ver, por un lado, las competencias interculturales que se han estado haciendo y, por otro lado, qué competencias intersociales son necesarias para afrontar el nuevo reto que surge para generar escuelas interculturales e intersociales. Dicho reto es el camino a marcar a partir de ahora cuando hablemos de enclaves educativos (ergo, sociedades) inclusivas y equitativas. Debemos posicionarnos en la necesidad de dichas competencias culturales y sociales, sólo así comprenderemos la diversidad desde una ecología de saberes, pero también desde la equidad de oportunidades, atendiendo la diversidad de diversidades.

\section{Financiación}

Este estudio es parte de una investigación posdoctoral financiada con una beca de excelencia otorgada por el Gobierno de México, a través de la Agencia Mexicana de Cooperación Internacional para el Desarrollo. 


\section{Referencias bibliográficas}

Barrett, M. et al. (2013). Developing Intercultural Competence throngh Education. Estrasburgo: Council of Europe. http://bit.ly/2NxOo7o

Baudelot, C., y Establet, R. (1976). La educación capitalista en Francia. Madrid: Siglo XXI. http://bit.ly/2pMoN1F

Baumann, G. (2001). El Enigma Multicultural. Un replanteamiento de las identidades nacionales, etnicas y religiosas. Barcelona: Paidos. http://bit.ly/2K1yyQg

Bonfil Batalla, G. (1995). Culturas populares y politica cultural. México, D.F.: Dirección General de Culturas Populares e Indígenas. https://bit.ly/2Zd5v55

Bourdieu, P., \& Passeron, J. (1977). Reproduction in society, education and culture. Trans. R. Nice. London: Sage. http://bit.ly/2p1rOdU

Borja, J. y Manuel Castells, y otros. (1998). Local y global: la gestión de las ciudades en la era de la información. Madrid: Taurus. https://doi.org/10.4067/s0250-71611998007300008

Byram, M., Nichols \& Stevens, D. (2001). Developing Intercultural Competence in Practice. Clevedon: Multilingual Matters. http://bit.ly/2CrAuNP

Camacho, G. M. (2006). Gramsci y el proceso hegemónico educativo. Revista Electrónica Educare, 9(2), 13-39. http://bit.ly/2NV8445. Doi: http://doi.org/10.15359/ree.9-2.1.

Dietz, G. (2011). Hacia una etnografía doblemente reflexiva: una propuesta desde la antropología de la interculturalidad. AIBR Revista de Antropología Iberoamericana, 6(1), 3-26. DOi: https://doi.org/10.11156/aibr.060102

Dietz, G. (2019) Competencia intercultural. En: Irlanda Villegas, Gunther Dietz \& Miguel FigueroaSaavedra (eds.): La traducción lingüistica y cultural en los procesos educativos: hacia un vocabulario interdisciplinar. Xalapa, Ver. - Ciudad de México: Editorial de la Universidad Veracruzana - Facultad de Filosofía y Letras de la UNAM [en prensa]

Enrico, L. (2008). Lectura, ciudadanía y educación: Miradas desde la diferencia. Revista Pilquen, (10), $0-0$. http://bit.ly/34Lx3xs

Lerena, A. (1975). Sistema de enseñanza y desarrollo capitalista en España (Tesis doctoral). Madrid: Facultad CCEE. Univ. Complutense.

Mato, D. coord. (2008). Diversidad cultural e interculturalidad en Educación Superior. Experiencias en América Latina. Caracas: IESALC-UNESCO. http://bit.ly/2NvPNeD

Ogbu, J. U., y Simons, H. D. (1998). Voluntary and involuntary minorities: a cultural-ecological theory of school performance with some implications for education. Anthropology \& Education Quarterly, 29(2), 155-188. Doi: https://doi.org/10.1525/aeq.1998.29.2.155

Otero, J. y Horcajo, F. (2011). Teoría y práctica de las competencias básicas. Barcelona: Graó. http://bit.ly/33yFBYp

Panaia, M.. (2010). Algunas precisiones sobre el concepto de población flotante en el ámbito del trabajo. Pampa, 6, 27-36. Doi:_https://doi.org/10.14409/pampa.v1i6.3175.

Portera, A. \& C.A. Grant (eds.). (2017). Intercultural Education and Competences: challenges and answers for the global world. Newcastle upon Tyne: Cambridge Scholars Publishing. http://bit.ly/2Nw8E9z

Rivero, J. (2002). La importancia de la educación en la determinación de la hegemonía: Las teorías de la reproducción. Filosofía, política y economía en el Laberinto, (8), 72-84. http://bit.ly/2X0hf7G

Santaolalla, P. (2016a). Modelo Educativo Discursivo: autoetnoecología e Investigación-Acción Participante en la zona norte de Alicante (Tesis doctoral). Elche, Alicante: Facultad de CCSS de la Universidad Miguel Hernández. http://bit.ly/2KM14bC

Santaolalla, P. (2016b). Educación en la periferia social: un engranaje diseñado para invisibilizar la exclusión. Revista Nuevas Tendencias en Antropologia, 7, 172-212. http://bit.ly/35rmYpL

Santaolalla, P. (2017). Proyecto Interculturalidad en barrios del PlaCarolinas, Campoamor y San Antón. Alicante: Concejalía de Inmigración del Ayuntamiento de Alicante. http://bit.ly/2Jg568R 
Santaolalla, P. (2018). Investigación Aplicada Implicada: metodologías para la transformación educativa-social. En M. H. Fernández-Carrión (Ed.), Educación actual. I Entre el pasado y el presente (pp. 57-77). Ciudad de México: CiECAL. https://amzn.to/37szA21

Torres, P. (1994). Democracia inacabada, transición o ficción democrática: los límites entre la reestructuración y el reformismo en América Latina. Nuevos itinerarios para un viejo problema. Papers: revista de sociologia, (44), 29-52. http://bit.ly/36QfTAy

Willis, P. (2017)[1978]. Learning to labour: How working class kids get working class jobs. London: Routledge. http://bit.ly/32uZZZk

\section{Lista de referencias web}

Consellería de Educación, Cultura y Deporte Comunidad Valenciana (2014-2017). [Estadísticas Educativas a nivel autonómico]. España: Ceice Gva. Recuperado de: http://bit.ly/2MHdQaj

Instituto Nacional Evaluación Educativa (2014-2017). Evaluaciones nacionales. España: Educaciónyfp INEE). Recuperado de: http://bit.ly/2N4CtwA

Servicios Educativos Quintana Roo (2018-2019). Solicitud de acceso a la información pública. México: Trámites y Gestión Pública. Recuperado de: http://bit.ly/2PfQ7j0

Consejo Nacional de Evaluación de la Política de Desarrollo Social (2018-2019): Medición de la pobreza. México: CONEVAL. Recuperado de: http://bit.ly/35TCqMF

Instituto Nacional de Información Estadística Educativa (2018). [Consulta de estadísticas educativas por Estado, escuelas e indicadores educativos. México: INEGI. Recuperado de: http://bit.ly/33XYwf7. En 2019 trasladado a diferentes órganos y webs: http://bit.ly/2Q3zTtG y http://bit.ly/2Q031SJ

Instituto Nacional de Estadística (2014-2017). Estadísticas territoriales. España: INE. Recuperado de: http://bit.ly/31F4nEB

Generalitat Valenciana (2014): [Servicio datos y estadísticas territoriales de Vivienda y Rehabilitación] España: Habitatge GVA. Recuperado de: http://bit.ly/31K9JOF

Ministerio de Trabajo, Migraciones y Seguridad Social de España (2014-2017). Estadísticas del Portal de Inmigración. España: Extranjeros Mitramiss. Recuperado de: http://bit.ly/2NbHOlw

\section{Transferencia}

Desde este texto nace una línea de investigación que seguiremos abordando desde lo intersocial, donde, de igual forma, se están elaborando patentes para materiales didácticos concretos en base a lo que hemos denominado como Inter-proyectos; además de un modelo educativo que aborda los ámbitos de equidad desde lo intercultural y lo intersocial y una metodología participativa en base a programas para escuelas sobre competencias intersociales que, a día de hoy, están siendo elaborados y puestos en práctica (para la comprobación de su viabilidad, limitaciones, repercusión sobre la sociedad, oportunidades, etc.) entre dos centros educativos (privado y público).

\section{Para citar este artículo}

Santaolalla Rueda, P. (2019). Lograr la equidad en educación a través de competencias interculturales e intersociales. Revista Fuentes, 21(2), 229-238. [Fecha de consulta: dd/mm/aa]. doi: 10.12795/revistafuentes.2019.v21.11.07 